\title{
Expression of pre-selected TMEMs with predicted ER localization as potential classifiers of ccRCC tumors
}

Tomasz Wrzesiński ', Malgorzata Szelag², Wojciech A. Cieślikowski ${ }^{3}$, Agnieszka Ida $^{3}$, Rachel Giles ${ }^{4}$, Elżbieta Zodro ${ }^{1}$, Joanna Szumska', Joanna Poźniak', Zbigniew Kwias³ ${ }^{3}$ Hans A.R. Bluyssen² and Joanna Wesoly ${ }^{1 *}$

\begin{abstract}
Background: VHL inactivation is the most established molecular characteristic of clear cell renal cell carcinoma (ccRCC), with only a few additional genes implicated in development of this kidney tumor. In recently published cCRCC gene expression meta-analysis study we identified a number of deregulated genes with limited information available concerning their biological role, represented by gene transcripts belonging to transmembrane proteins family (TMEMs). TMEMs are predicted to be components of cellular membranes, such as mitochondrial membranes, ER, lysosomes and Golgi apparatus. Interestingly, the function of majority of TMEMs remains unclear. Here, we analyzed expression of ten TMEM genes in the context of cCRCC progression and development, and characterized these proteins bioinformatically.

Methods: The expression of ten TMEMs (RTP3, SLC35G2, TMEM30B, TMEM45A, TMEM45B, TMEM61, TMEM72, TMEM116, TMEM207 and TMEM213) was measured by qPCR. T-test, Pearson correlation, univariate and multivariate logistic and Cox regression were used in statistical analysis. The topology of studied proteins was predicted with Metaserver, together with PSORTII, Pfam and Localizome tools.

Results: We observed significant deregulation of expression of 10 analyzed TMEMs in ccRCC tumors. Cluster analysis of expression data suggested the down-regulation of all tested TMEMs to be a descriptor of the most advanced tumors. Logistic and Cox regression potentially linked TMEM expression to clinical parameters such as: metastasis, Fuhrman grade and overall survival. Topology predictions classified majority of analyzed TMEMs as type 3 and type 1 transmembrane proteins, with predicted localization mainly in ER.

Conclusions: The massive down-regulation of expression of TMEM family members suggests their importance in the pathogenesis of cCRCC and the bioinformatic analysis of TMEM topology implies a significant involvement of ER proteins in CCRCC pathology.
\end{abstract}

Keywords: ccRCC, Transmembrane proteins, ER, Gene expression, Bioinformatics

\section{Background}

Renal cell carcinoma (RCC) is a relatively common malignancy accounting for approximately $2 \%$ of all adult cancers and causing approximately 100,000 deaths per year worldwide [1]. $80 \%$ of renal cell carcinoma (RCC) cases are classified as clear cell renal cell carcinoma (ccRCC), originated

\footnotetext{
*Correspondence: j.wesoly@amu.edu.pl

'Laboratory of High Throughput Technologies, Institute of Molecular Biology and Biotechnology, Faculty of Biology, Adam Mickiewicz University, Umultowska 89, 61-614 Poznan, Poland

Full list of author information is available at the end of the article
}

from proximal convoluted tubule, with annually 168,000 newly diagnosed cases [2]. During last 30 years the incidence of RCC has been steadily increasing, likely due to the higher exposition to risk factors such as cigarette smoking, obesity, hypertension and accidental diagnosis due to improved visualization techniques [3].

Familial, associated with von Hippel-Lindau (VHL) syndrome, and sporadic ccRCC have been linked to inactivation of $V H L$ gene by mutation, loss of heterozygosity (LOH) and promoter hypermethylation [4-6]. VHL inactivation is the most established cause of ccRCC, but 
there are several other factors involved in pathogenesis of tumor type exemplified by mutations in KNG1 and MT1A genes [7], as well as PI3K/AKT/mTOR signaling pathway factors (MTOR, PTEN, PIK3CA) [8].

One group of ccRCC deregulated genes contains gene transcripts belonging to TMEM (transmembrane proteins) gene family. Differential regulation of TMEMs could be observed in many cancers, such as lymphomas (TMEM176) [9], colorectal cancer (TMEM25) [10], meningiomas (TME $M 30 B)$ [11], paragangliomas and pheochromocytomas (TM EM127) [12]. In case of ccRCC, up- or down-regulation of different TMEMs is supported by a number of microarray experiments [13-19].

In our recently published microarray-based meta-analysis, we identified a number of up- and down-regulated genes with limited information available concerning their biological role. We found 10 significantly deregulated genes $(8$ down- and 2 up-regulated) which were represented by gene transcripts belonging to TMEM gene family, with highest down-regulation of TMEM213 (effect size $=-11.7$ ) and highest up-regulation of TMEM45A (effect size $=2.6$ ) [20]. TMEMs are a group of ca. 310 different proteins (with ca. 440 identified isoforms and ca. 580 transcript variants) predicted to be components of cellular membranes, such as mitochondrial membranes, lysosomes and Golgi apparatus. Interestingly, the function of majority of TMEM proteins remains unclear, mainly due to difficulties in extraction and purification of transmembrane proteins. Nevertheless many TMEM proteins have been functionally assigned as transmembranous anion channels (e.g., ANO1) [21] and molecules responsible for oncosis (TMEM123) [22], protein glycosylation (TMEM165) [23], pathogen intoxication (TMEM181) [24], as well as innate immunity response (TMEM173) [25]. Although down-regulation of TMEMs has been detected in large number of microarrays on ccRCC tumors, not much is known about their function in pathogenesis of ccRCC.

Research performed by Kholodnyuk et al. showed that RTP3 (TMEM7) expression in 5 different RCC cell lines - KH39, CAKI-1, CAKI-2, KRC/Y and TK-10 was impaired in comparison to normal human kidney by RTPCR [26] and was suggested to act as of tumor suppressor gene. Additionally, overexpression of SLC35G2 (TMEM22) and RAB37 genes in ccRCC tumors was observed $[27,28]$ and experiments on RCC cell lines implied that the SLC35G2/RAB37 complex was likely to play a crucial role in growth of RCC [29].

Here we compared expression of 10 TMEM family members: RTP3, SLC35G2, TMEM30B, TMEM45A, TME M45B, TMEM61, TMEM72, TMEM116, TMEM207 and TMEM213 in tumors histopathologically classified as ccRCC. Additionally, we correlated expression of these genes with VHL, HIF1A and EPAS1 expression. We also analyzed expression of 10 TMEM genes in PBMCs of patients with metastatic and non-metastatic ccRCC, at the time of nephrectomy and a year post-nephrectomy. Moreover, we tested if expression of TMEM could be utilized as a potential predictor for metastases, progression-free disease course and patient survival rate. Lastly, we tried to predict topology and tertiary structure of TMEMs using bioinformatic tools.

\section{Methods}

\section{Patient material collection}

The samples were obtained prospectively from 76 histopathologically confirmed ccRCC tumors (Additional file 1 : Table S2). Collected tissue represented cross-section of kidney tissue (i.e., inner tumor mass, border of a tumor and non-tumoral kidney tissue). Analyzed tissue represented inner but not necrotic tumor tissue. Tissues were suspended in RNALater reagent (Sigma-Aldrich, St. Louis, MO, USA). Peripheral blood from ccRCC patients was collected before nephrectomy and ca. a year post surgery (Additional file 2: Table S3). Mean time of a followup was equal to 13.31 months, with median 12.5 months and range 3-34 months. Tissues and PBMCs (Peripheral Blood Mononuclear Cells) samples were obtained from the Department of Urology and Urological Oncology, Poznan University of Medical Sciences. This research was approved by Local Bioethical Committee at Poznan University of Medical Sciences (Poland), no. 1124/12 and written informed consent to participate in the study and to publish individual clinical data (including age and gender) was obtained from all patients. Disease progression was defined as local neoplasm recurrence or distant metastasis detected by at least one of following methods: Xray, abdominal ultrasound and computer tomography of chest and abdomen. Detailed patient clinical characteristics are presented in Table 1.

\section{RNA isolation from tissues and PBMCs}

Tissue total RNA was isolated from homogenized tumor tissue using GeneMATRIX Universal RNA Purification Kit (EurX, Gdańsk, Poland) following supplied protocol. PBMC RNA was isolated using Ficoll gradient (GE Healthcare, Little Chalfont, Buckinghamshire, England) followed by RNA extraction using TRI Reagent ${ }^{\circ}$ (Molecular Research Center, Cincinnati, OH, USA). RNA quality and quantity was determined with NanoDrop Spectrophotometer ND-1000 (Thermo Scientific, Wilmington, DE, USA).

\section{Reverse transcription and real-time PCR}

$1 \mu \mathrm{g}$ of RNA was reversely transcribed using RevertAid ${ }^{\mathrm{mx}}$ First Strand cDNA Synthesis Kit with Random Hexamers (Thermo Scientific Fermentas, Waltham, MA, USA) following supplied protocol.

Analyses were performed using Eco Real Time System (Illumina, San Diego, CA, USA), with Maxima ${ }^{\text {Tw }}$ SYBR 
Table 1 Characterization of ccRCC patient cohort. Median age of healthy donors which PBMCs were obtained from was 42.50 years old, $25 \%$ Percentile $=35,75 \%$ Percentile $=46.75$. Abbreviations: NM - not measured

\begin{tabular}{|c|c|c|c|}
\hline \multirow{2}{*}{$\begin{array}{l}\text { Name of the } \\
\text { characteristic }\end{array}$} & \multicolumn{3}{|l|}{ Amount } \\
\hline & Tumor tissue & PBMCs before nephrectomy & PBMCs 12 months post-nephrectomy \\
\hline \multicolumn{4}{|c|}{ Number of samples included in the study } \\
\hline Patients & 76 & 66 & 27 \\
\hline Controls & 23 & 14 & 14 \\
\hline \multicolumn{4}{|c|}{ Gender } \\
\hline Males (\%) & $45(59)$ & $45(68)$ & $19(70)$ \\
\hline Females (\%) & $31(41)$ & $21(32)$ & $8(30)$ \\
\hline \multicolumn{4}{|c|}{ Age (at a time of surgery) } \\
\hline Median & 64 & 65 & 61 \\
\hline $25 \%$ Percentile & 57 & 56 & 53 \\
\hline $75 \%$ Percentile & 72 & 73 & 69 \\
\hline \multicolumn{4}{|c|}{ Tumor size $[\mathrm{mm}]$} \\
\hline Median & 43 & 43.50 & 44 \\
\hline $25 \%$ Percentile & 33 & 31.88 & 28.50 \\
\hline $75 \%$ Percentile & 65.38 & 65 & 59 \\
\hline \multicolumn{4}{|c|}{ TNM stage - pT } \\
\hline pT1 (\%) & $40(52)$ & $34(51)$ & $16(59)$ \\
\hline pT2 (\%) & $2(3)$ & $3(5)$ & $2(8)$ \\
\hline pT3 (\%) & $32(42)$ & $28(42)$ & $9(33)$ \\
\hline pT4 (\%) & $2(3)$ & $1(2)$ & $0(0)$ \\
\hline \multicolumn{4}{|c|}{ TNM stage - pN } \\
\hline pNo (\%) & $73(96)$ & $63(95)$ & $27(100)$ \\
\hline pN1 (\%) & $3(4)$ & $3(5)$ & 0 \\
\hline \multicolumn{4}{|c|}{ TNM stage - M } \\
\hline M0 (\%) & $62(82)$ & $52(79)$ & $25(93)$ \\
\hline M1 (\%) & $14(18)$ & $14(21)$ & $2(7)$ \\
\hline \multicolumn{4}{|c|}{ Fuhrman grade } \\
\hline G1 (\%) & $4(6)$ & $4(6)$ & $0(0)$ \\
\hline G2 (\%) & $35(46)$ & $32(48)$ & $17(63)$ \\
\hline G3 (\%) & $26(34)$ & $22(33)$ & $9(33)$ \\
\hline G4 (\%) & $11(14)$ & $8(13)$ & $1(4)$ \\
\hline \multicolumn{4}{|c|}{ VHL expression } \\
\hline VHL+ & 33 & NM & NM \\
\hline VHL- & 43 & NM & NM \\
\hline
\end{tabular}

Green/ROX qPCR Master Mix (2×) (Thermo Scientific Fermentas, Waltham, MA, USA) following supplied protocol. Primers were designed using PrimerBLAST (http://www.ncbi.nlm.nih.gov/tools/primerblast) and Oligo Analyzer 3.1 (http://eu.idtdna.com/ analyzer/applications/oligoanalyzer/default.aspx). Analyzed samples were corrected by reaction efficiency obtained from standard curves which varied from $91 \%$ to $110 \%$. Primers used for the analyses are listed in Additional file 3: Table S1. Expression of each gene in ccRCC tissue samples and PBMCs was measured in duplicates, in two independent experiments. Calculated normalized relative quantities (CNRQ) of transcripts were obtained by normalization to reference gene $(A C T B)$ and average of reference samples using qBase Plus software (https://www.biogazelle.com/qbaseplus).

\section{Statistical analysis}

All charts were prepared using GraphPad Prism software v6.02 (http://www.graphpad.com). IBM SPSS Statistics 
software v22 (http://www-01.ibm.com/software/analytics/spss/products/statistics/) was used for $t$-test, ANOVA and Pearson correlations. For clusters analysis TMEM expression values were dummy-coded basing on their deviation from average expression among all tumor samples. Univariate and multivariate logistic regression, and univariate and multivariate Cox regression were performed using $\mathrm{R}$ statistical software v3.1.1 [30]. For regression models, AIC (Akaike information criterion) [31] was used to assess the relative quality of each predictor, as compared to the null model. Firstly, in case of multivariate logistic regression, maximum 4 best predictors was chosen (to prevent a model from overfitting) based on best subset selection using leaps package [32] and the lasso shrinkage method using glmnet package [33, 34]. Each variable were tested for colinearity using variance inflation factor (VIF) calculation [35]. Then, each coefficient was recalculated using penalized maximum likelihood estimation to avoid overfitting of a model by rms package [36-40]. To assess true standard error and prediction capabilities of each model bootstrap and cross-validation methods were used, as implemented in rms library [37]. Univariate Cox regression to assess the marginal effect of each factor (when not correcting for the effect of other factors) were performed. Multivariate Cox regression was calculated by rms package and variables in the model were included based on step-wise method and the lasso shrinkage method using glmnet package [41]. Each variable included in Cox regression model was tested for proportional hazards assumption based on Grambsch and Therneau method [42]. All pvalues obtained during statistical analyses (i.e., from $\mathrm{t}$ tests, ANOVA, Pearson correlations, univariate and multivariate logistic and Cox regression models) were adjusted all together for multiple comparisons using Benjamini-Hochberg method [43], and obtained q-values were considered to be statistically significant at $\mathrm{q} \leq 0.1$.

Bioinformatic prediction of TMEMs topology and function Query sequences selection: The following NCBI RefSeq sequences of native human TMEMs were used for bioinformatics analysis, NCBI GIs: 146229352 (TMEM213), 46409276 (TMEM207), 32698902 (TMEM61), 20270331 (TMEM45B), 63003930 (TMEM30B), 13899263 (RTP3), 183227675 (TMEM72), 302058299 (TMEM116), 14822 4156 (SLC35G2) and 8922243 (TMEM45A). Pfam analysis: Query sequences were submitted to the Pfam 27.0 database of protein families and Hidden Markov model HMM searches (default Pfam-A data set and e-value of 1.0) were conducted [44]. Fold recognition analysis Metaserver: Full-length sequences were submitted to Genesilico Metaserver gateway [45]. The consensus of different programs and servers was used to determine transmembrane helices, protein order and secondary structure, using 12,
16 and 17 methods, respectively. Protein solvation prediction was determined with 6 different programs and relative solvent accessibility of amino acid (RSA, \%). An aa was considered buried with RSA value lower than a assumed, implemented threshold ( $25 \%, 5 \%$, or $0 \%)$. If RSA value for the aa was higher than the threshold, it was considered as exposed. TMEM homologs with tertiary structures deposited in RCSB PDB were determined by foldrecognition methods, which were compared, evaluated, and ranked by the Pcons5 algorithm [46]. PSORT II: Sequences were submitted and analyzed based on their amino acid sequence [47]. The presence of signal sequences was determined by recognizing positively charged $\mathrm{N}$-region, the central hydrophobic region and searching the position of possible cleavage site [48, 49]. TM segments were predicted with ALOM method and the membrane topology was scored using Singer's classification $[50,51]$. The cytoplasmic/nuclear discrimination for TMEMs was analyzed by Reinhardt's algorithm, which determines the probability of protein localization by ' $\mathrm{NNCN}$ score` [52]. Localization assignment: mitochondrial [47], nuclear [52], peroxisomal [53], endoplasmic reticulum [54], lysosomal and vacuolar [55] were presented as consensus with the use of k-nearest neighbor (k-NN) algorithm [56]. Localizome: TM helix number and topology was predicted with Phobius algorithm [57, 58]. The hmmpfam method was employed (default e-value of 0.01 ) to include domains obtained from Pfam. Combined results were validated by LocaloDom database of protein domains. Consensus analysis: Metaserver, PSORT II and Localizome predictions of TMEMs were superimposed. Obtained colocalization of the data was analyzed to find the most probable protein topology and evaluate the reliability of the calculations.

\section{Results \\ TMEM expression in tumor tissue}

In line with our previous observations, we detected statistically significant differences in expression of all ten TMEMs in tumor tissue and healthy kidney tissue (Fig. 1a) [20]. The down-regulation varied between the genes, with 1758.34 fold for TMEM213 (q < 0.0001) and 3.06 fold for TMEM116 (q<0.05). Both, SLC35G2 and TMEM45A were found to be up-regulated with $5.33(\mathrm{q}<0.01)$ and 6.75 fold change $(\mathrm{q}<$ $0.0001)$, respectively. We investigated if analyzed TMEMs displayed differential expression in metastatic and nonmetastatic tumors. As shown in Fig. 1b, we detected significant difference in expression of TMEM72 and TMEM116: 39.78 fold and 6.65 fold down-regulation in metastatic tissue ( $n=12$ and $n=14$, respectively) as compared to 5.13 fold and 2.57 fold in the non-metastatic samples $(n=58, \mathrm{q}=0.051$ and $n=62, \mathrm{q}=0.056$, respectively). Next, we subdivided samples into organ-confined tumors $(\mathrm{pT} \leq 2)$ and advanced tumors $(\mathrm{pT} \geq 3)$. A decrease 


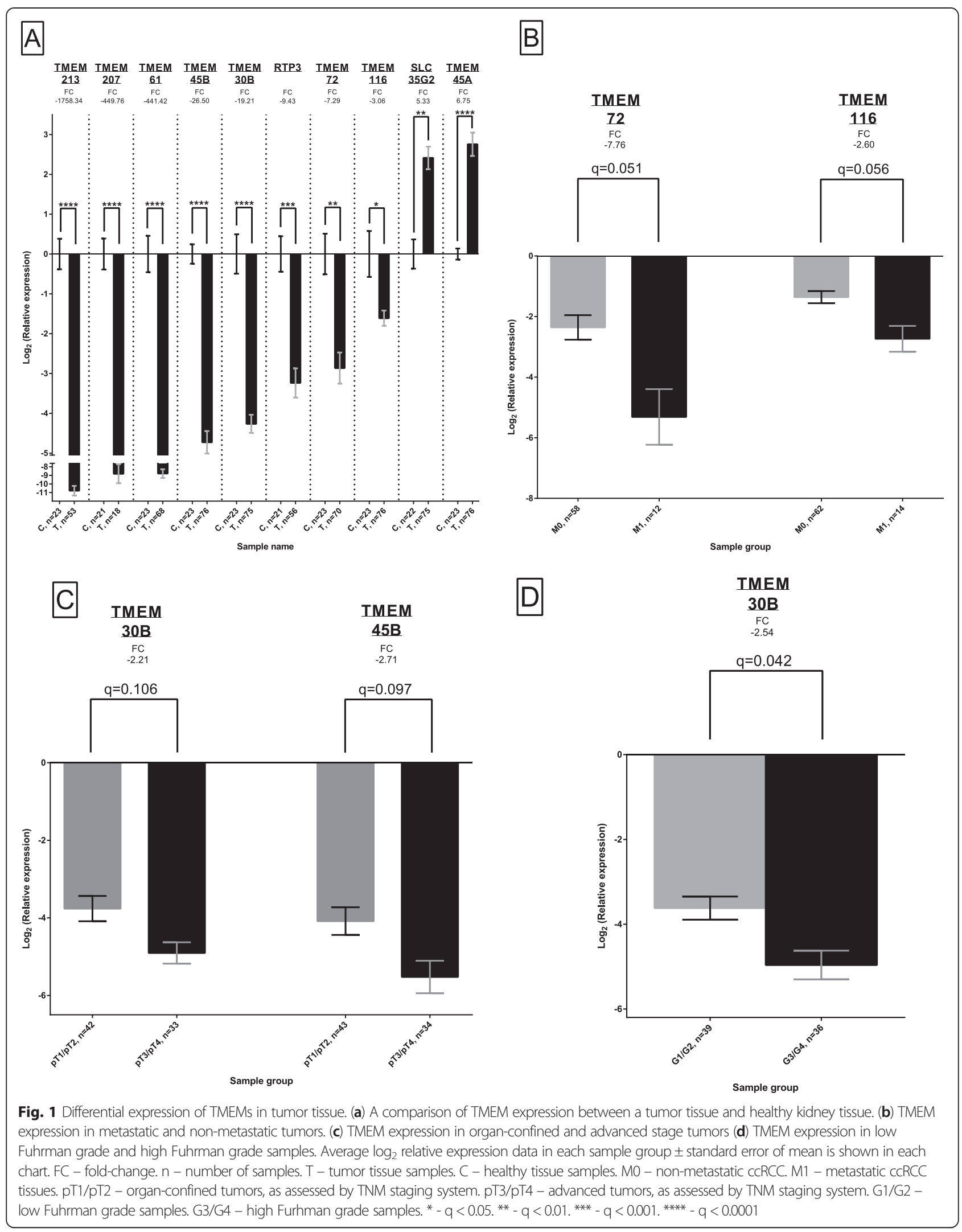


of TMEM30B ( $\mathrm{q}=0.106)$ and TMEM45B $(\mathrm{q}=0.097)$ expression was found in advanced-stage samples, with -2.21 and -2.71 fold-change, as compared to early-stage tumors, respectively (Fig. 1c). T-test was used to compare TMEM expression in well differentiated tumors (Fuhrman grade, $G \leq 2)$ with undifferentiated tissues $(G \geq 3$, Fig. $1 d)$ and we observed a stronger down-regulation of TMEM3OB in high Fuhrman grade samples (fold-change -31.18, $n=36$ ) in contrast to low Fuhrman grade samples (fold-change $-12.29, n$ $=39, \mathrm{q}<0.05)$. Expression comparisons of all analyzed 10 TMEM genes in metastatic and non-metastatic tumors, early- and late stage samples, and tumors with undifferentiated and well-differentiated tissues are shown in Additional file 4, 5 and 6: Figures S1-S3, respectively.

Pearson correlation of TMEMs and VHL, HIF1A and EPAS1 expression values was performed but correlation coefficient did not exceed 0.55 (in case of EPAS1 expression correlated with SLC35G2 $(\mathrm{r}=0.55)$, TMEM3OB $(\mathrm{r}=$ 0.32), TMEM72 $(\mathrm{r}=0.44)$ and TMEM116 $(\mathrm{r}=0.35)$, data not shown). Finally, we correlated TMEM expression between each other and overall we found the highest statistically significant Pearson correlation coefficient in case of TMEM213 with TMEM30B (Fig. 2a) and for TMEM72 correlated with TMEM116 (Fig. 2b).

In attempt to stratify the ccRCC tumors and to establish common expression profile based on TMEM expression we performed hierarchical clustering of available data (Fig. 3). TMEM expression values were dummy-coded basing on their deviation from average expression among all tumor samples as samples with TMEM expression above average (coded as " +1 ") and below average (coded as "-1"). Using IBM SPSS Statistics v22 software we identified five distinctive tumor clusters. Cluster I was characterized by low expression of TMEM72, TMEM116, TMEM207 and TMEM213. Samples with low expression of RTP3, TMEM207 and TMEM213 and with high expression of SLC35G2, TMEM72 and TMEM116 were preferentially assigned to cluster II. In cluster III low expression of TMEM $45 A$ and TMEM72, and high expression of TMEM61 were observed. Low expression of TMEM30B, TMEM207 and high expression of RTP3, TMEM45A and TMEM116 were distinctive for cluster IV. Cluster V was characterized by high expression of TMEM $30 B, T M E$ $M 45 B$ and TMEM213. Interestingly, cluster I was overrepresented by samples with more advanced disease stage $(n=21)$, as measured by tumor stage (TNM scale), Fuhrman grade and metastasis and cluster $\mathrm{V}$ contained less advanced tumors.

TMEM expression in tumor tissue as independent predictors of metastases, Fuhrman grade and disease progression

We checked TMEM expression in subgroups divided according to clinical parameters (i.e., gender, smoking status,

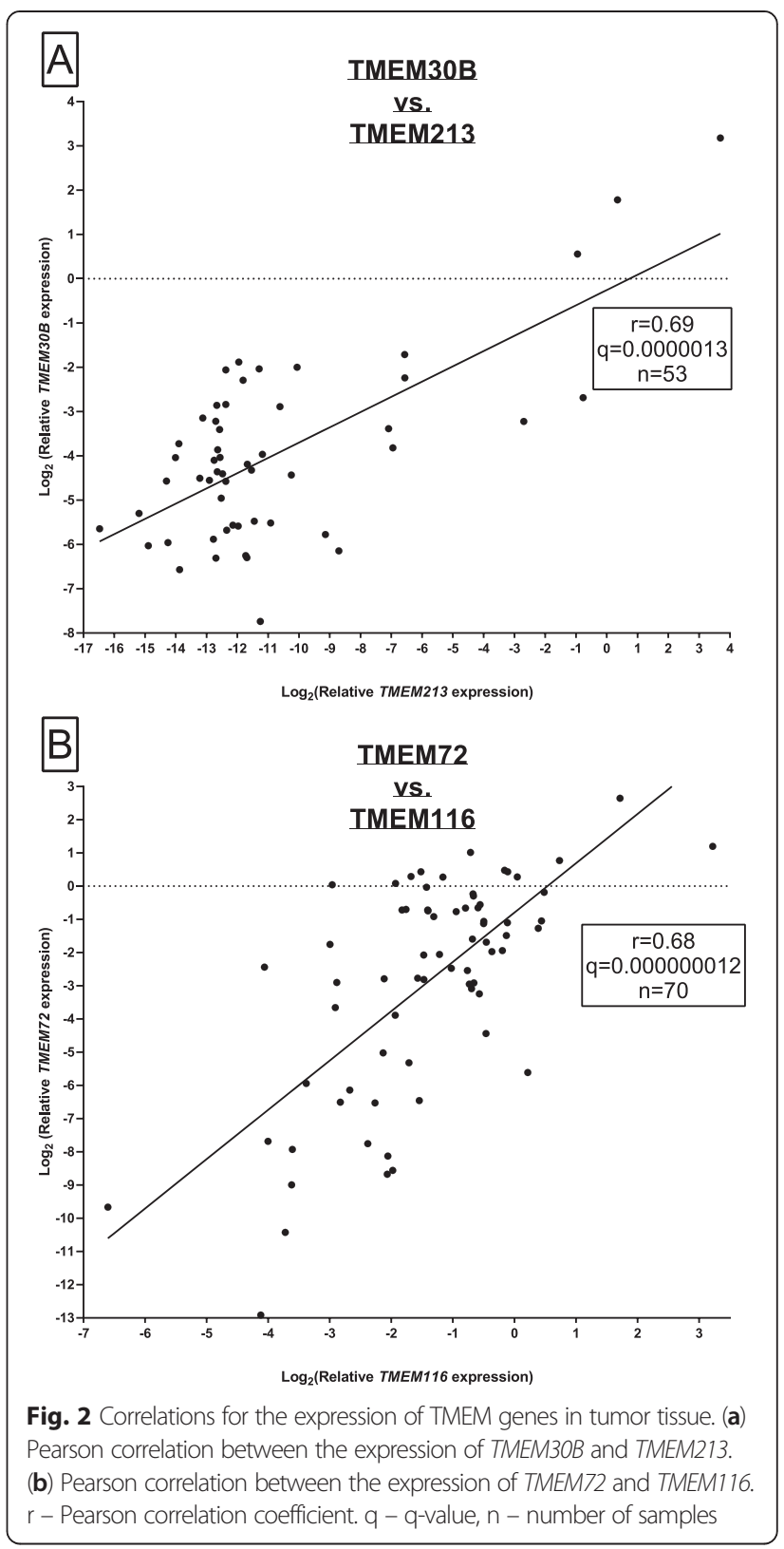

hypertension etc.) and analyzed TMEM correlation with continuous variables, such as age, average tumor size etc., but we did not find any significant differences (data not shown).

To evaluate the independent prediction of TMEM expression for clinical parameters univariate and multivariate logistic regression analyses were implemented (Table 2).

To evaluate differential TMEM expression as potential predictor of distant metastases univariate logistic regression was performed. It revealed that, together with average tumor size, presence of symptoms, and expression of EPAS1, 2-fold increase in the expression of TMEM72 and TMEM116 decreased the odds of metastases significantly for $22 \%$ and $40 \%$, respectively (Table $2 \mathrm{~A}$ ). Furthermore, 


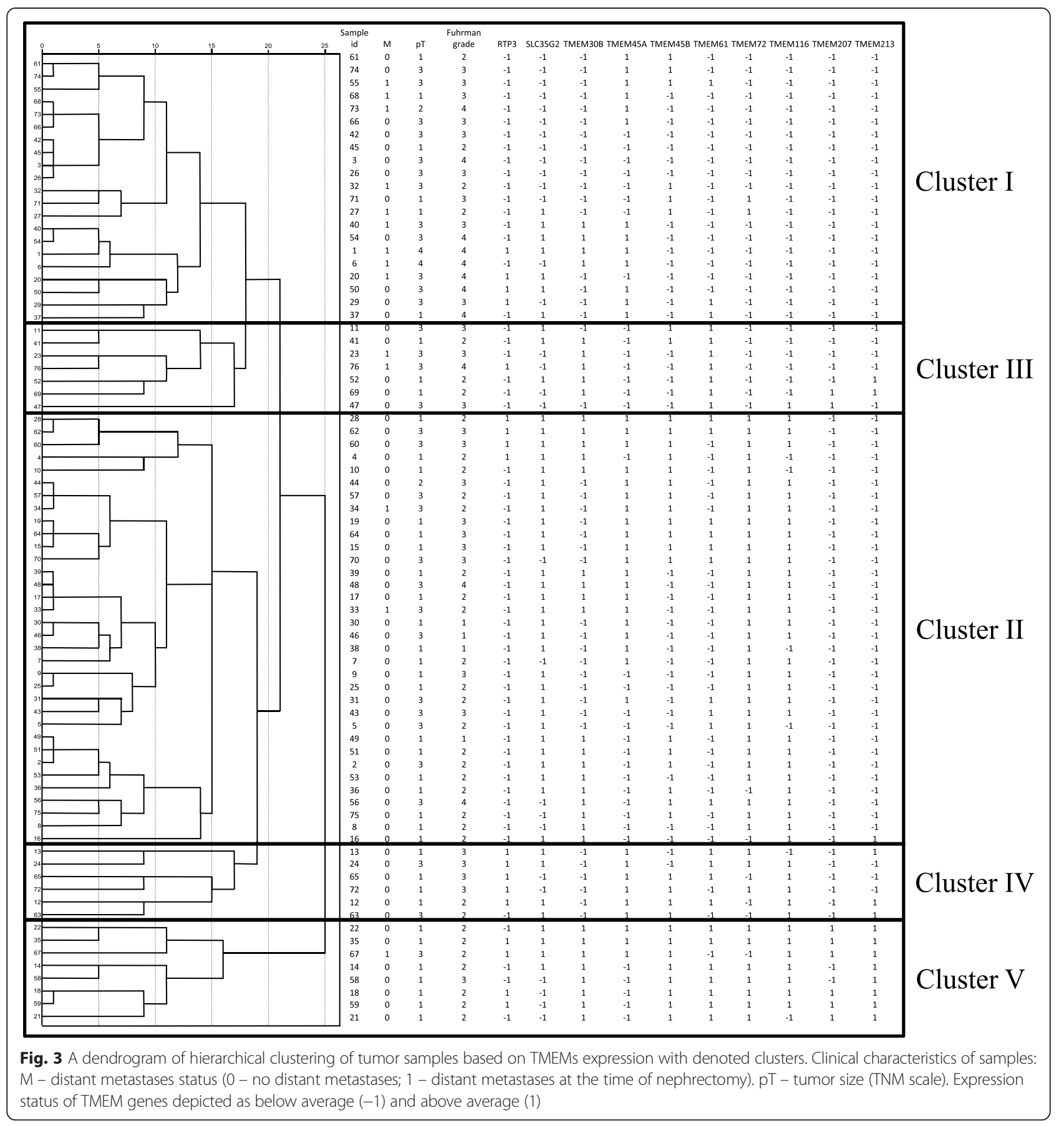

AIC of each TMEM predictor in univariate logistic regression was lower (60.67 and 68.84 for TMEM72 and TMEM116 expression respectively) than AIC of null model $(\mathrm{AIC}=74.61)$ suggesting potential predicting ability of analyzed variables. Removal of each of parameters (i.e., average tumor size in $\mathrm{mm}$ and TMEM72 relative expression) from multivariate model lowered the prediction ability of the model, as measured by likelihood ratio test ( $\mathrm{q}<$ 0.1 ). There were no collinear variables which could negatively influence prediction ability of the model, as assessed by VIF. The model was of good predictive ability as measured by ROC AUC values and $R^{2}$ (ROC AUC $=0.919$ and $0.932, R^{2}=0.565$ and 0.800 , measured by bootstrap and cross-validation methods correspondingly).

TMEM $30 B$ expression could be associated with significant decrease in odds of having high Fuhrman grade tumor in univariate logistic regression analysis (Table 2B). It had a potential predicting ability in case of Fuhrman grade classification, as measured by AIC (TMEM30B expression $\mathrm{AIC}=97.98)$ compared to null model $(\mathrm{AIC}=$ 
Table 2 Univariate and multivariate logistic regression analysis with clinical parameters and profiles of TMEM expression in tumors. (A) metastatic and non-metastatic samples. (B) Low and high Fuhrman grade samples. Confidence intervals and p-values in multivariate logistic regression were calculated using 10000-times bootstrap and were reported for penalized model. ROC AUC and R² parameters from both bootstrap and cross-validation were reported. Abbreviations: OR - odds ratio. $95 \%$ Cl OR - $95 \%$ confidence interval. AIC - Akaike Information Criterion. $\mathrm{n}$ - number of samples. ROC AUC - receiver operating characteristics area under curve parameter. VIF - variance inflation factor

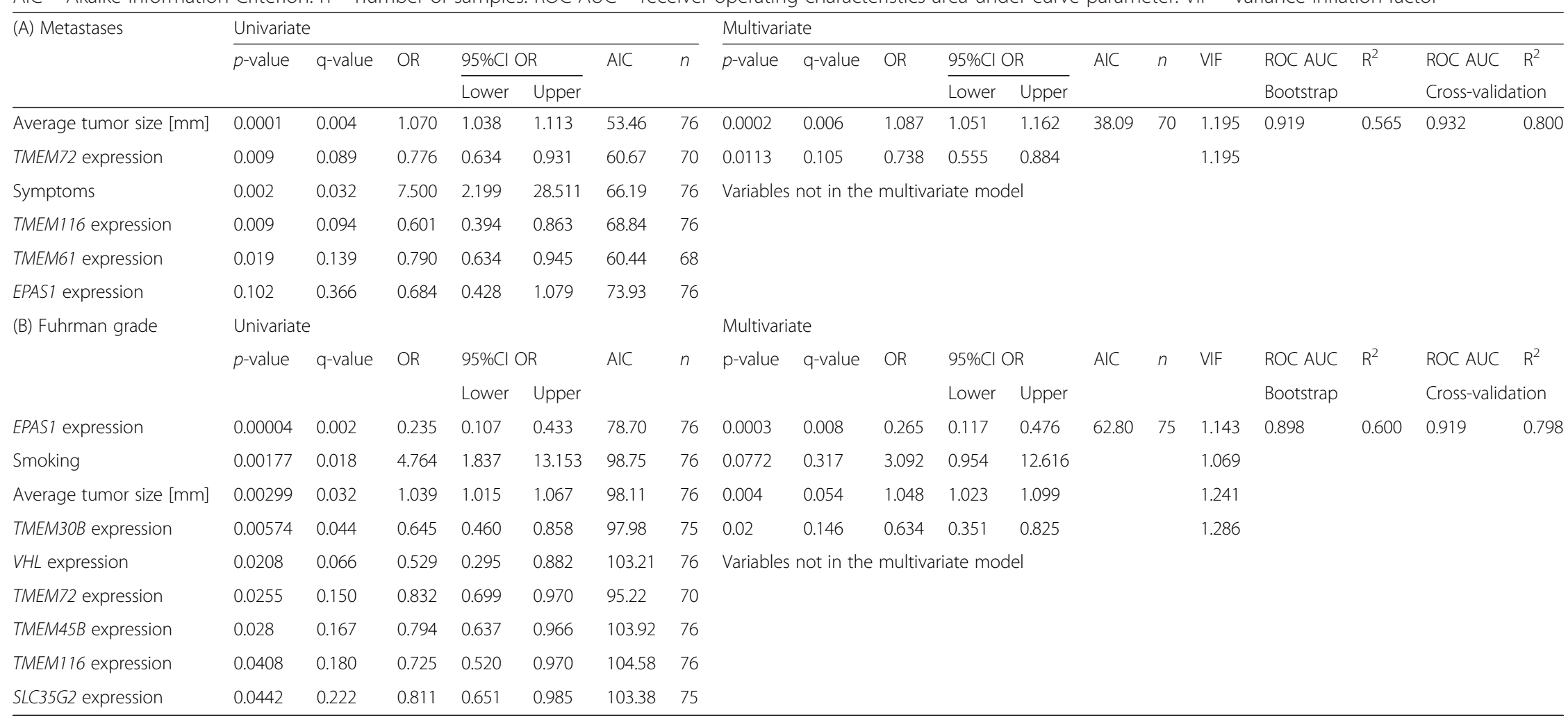


107.31). The multivariate logistic regression revealed that 2-fold up-regulation of TMEM3OB could be associated with significant decrease in odds of having high Fuhrman grade tumor by $37 \%$, in line with univariate logistic regression. Although TMEM3OB expression status did not reach statistical significance $(q>0.1)$, removal of each parameter from the model, would worsen the model significantly, as seen using likelihood ratio test $(\mathrm{q}<0.1)$. Bootstrapped and cross-validated predictive ability of the model was very good (ROC AUC $>0.89, R^{2} \geq 0.6$ ).

Univariate Cox regression (Table 3 ) revealed that, together with other factors, 2-fold increase of TMEM116 expression in tumor tissues is correlated with decreased risk of disease-related mortality (hazard ratio $=0.554, \mathrm{q}=$ 0.086). Although hazard ratio of TMEM72, SLC35G2 and TMEM61 expression were not statistically significant, AIC for these genes were lower (AIC $=24.52,48.63$ and 32.23, respectively) than the null model (AIC $=26.35$ for samples expressing TMEM72, $\mathrm{AIC}=49.45$ for samples expressing SLC35G2 and AIC $=34.47$ for tumors expressing TME M61 above detection limit). Multivariate regression model was built to assess TMEM expression as independent predictors of ccRCC-linked mortality but probably due to small sample size (total $n=76$, cases $=7$, censored $=59$, no follow-up $=10$ ) none of predictors was associated independently with this parameter $(\mathrm{q}>0.1)$. Progression-free survival was also assessed by univariate Cox regression and it revealed that expression of analyzed TMEMs could not be associated with progression-free disease course (q $>0.1$, data not shown).

\section{TMEM expression in PBMCs of ccRCC patients}

To assess the utility of TMEM expression as potential biomarkers we analyzed their expression in PBMCs of ccRCC patients (see Table 1). According to the Illumina Human Body Map 2.0 project (NCBI Gene Expression Omnibus accession no. GSE30611) six out of ten selected TMEMs were found to be expressed in PBMCs and those were included in further analysis (i.e., SLC35G2, TMEM30B, TMEM45A, TMEM45B, TMEM 116 and TMEM213). Although a comparison between expression of TMEMs in PBMCs obtained from patients before nephrectomy (T0) and from healthy volunteers did not reveal statistically significant differences, we observed significant down-regulation of TMEM213 (foldchange $=-12.24, \mathrm{q}<0.0001, n=17)$, down-regulation of TMEM45B (fold-change $=-2.47, \mathrm{q}<0.1, n=26$ ) and upregulation of $S L C 35 G 2$ (fold-change $=2.57, \mathrm{q}<0.1, n=25$ ) between PBMCs of healthy volunteers and PBMCs obtained from patients one year post nephrectomy (T2, Fig. 4). Furthermore, we found statistically significant down-regulation of TMEM213 and up-regulation of SLC35G2, as comparing time points before (T0) and 12 months after the surgery (T2), suggesting fluctuation of their expression in PBMCs post-nephrectomy (Fig. 4). No correlation between TMEM expression in tumors and PBMCs was observed (data not shown). Logistic regression did not reveal the dependence of clinical parameters (i.e., tumor size, Fuhrman grade, metastases and progression status) on TMEM expression in PBMCs (data not shown). Univariate Cox regression did not suggest TMEM's expression to be involved in disease progression or survival.

\section{Bioinformatic analyses of transmembrane proteins}

In order to determine the topology and structure of 10 TMEMs, found deregulated on the mRNA level in ccRCC tumors, extensive bioinformatic structural analysis was performed. Query sequences from NCBI were submitted to Pfam to classify TMEMs into known protein families [44]. Moreover, three independent protein structure prediction gateways: Metaserver [45], PSORT II [47] and Localizome [57] (Table 4) were used to find consensus of the proteins topology with Pfam results.

TMEM213 was the most down-regulated gene of all TMEMs tested in ccRCC samples. According to Pfam

Table 3 Univariate and multivariate overall survival Cox regression model with clinical parameters and profiles of TMEM expression in tumors. Total cases $=66$, censored cases $=59$. Abbreviations: HR - hazard ratio. $95 \% \mathrm{Cl} \mathrm{HR}-95 \%$ confidence interval. AlC - Akaike Information Criterion. $\mathrm{n}$ - number of samples. $\mathrm{n}$ events - number of deaths (i.e., cases not censored). VIF - variance inflation factor

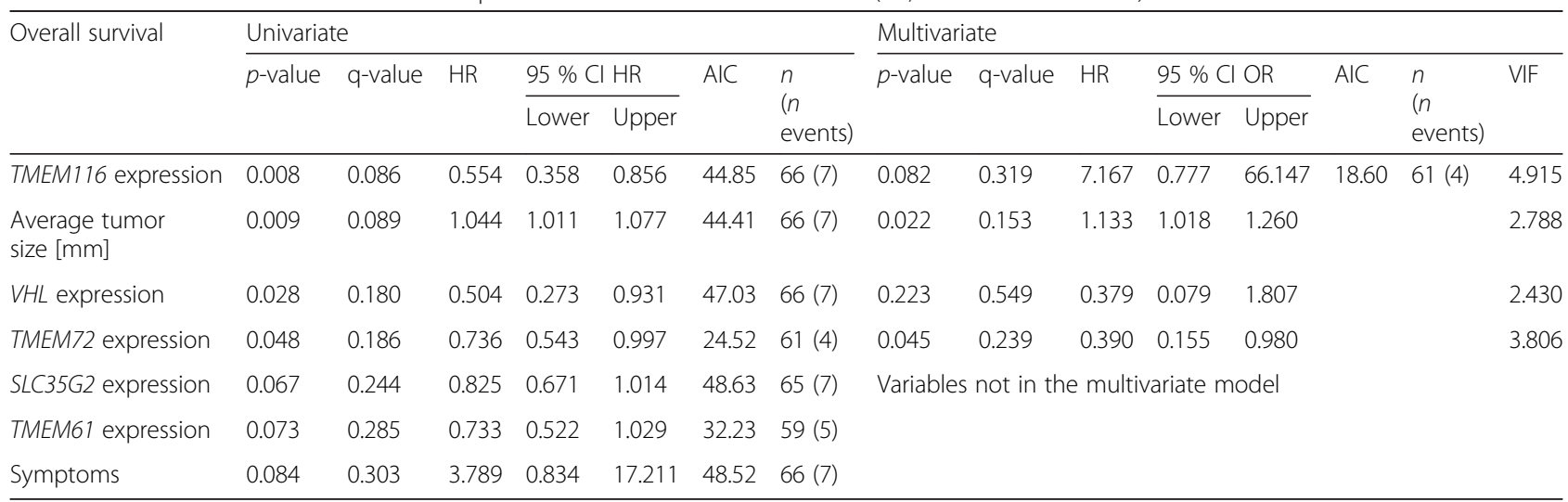




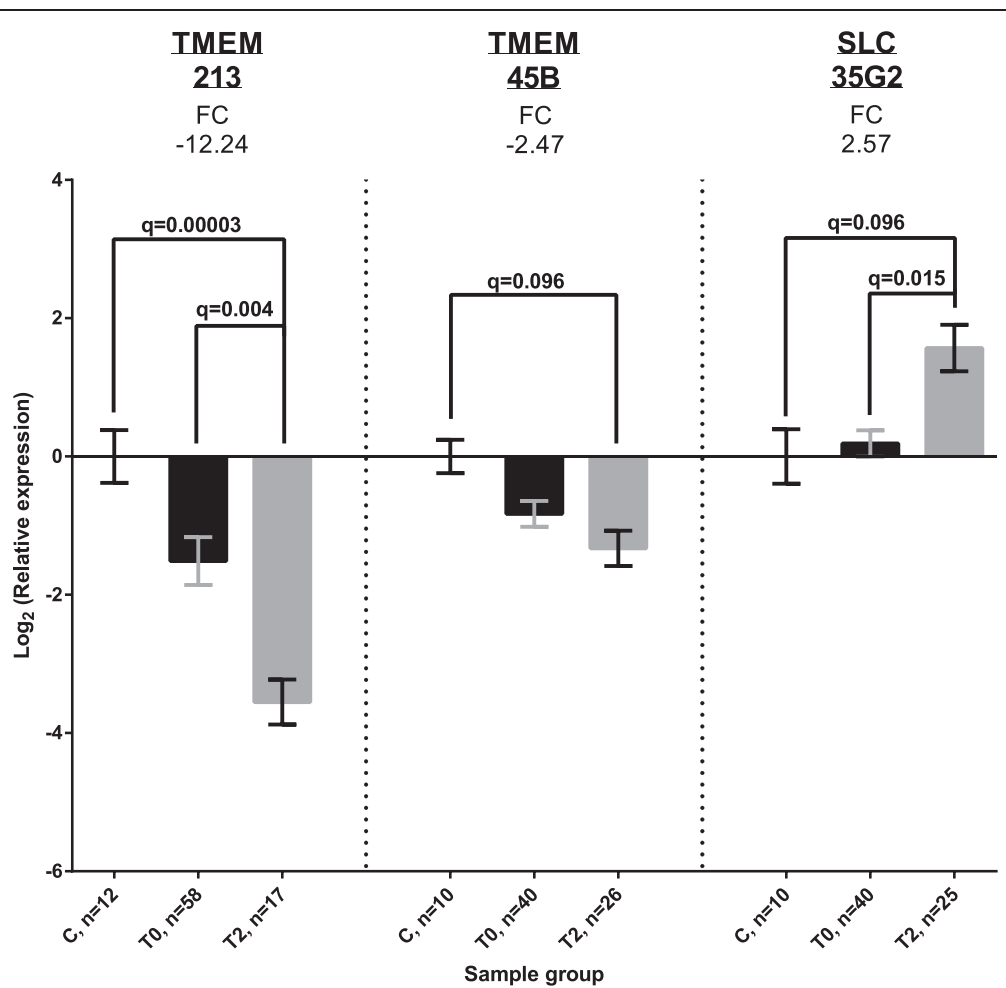

Fig. 4 A comparison of TMEM expression in PBMCs. Average $\log _{2}$ relative expression data in each sample group \pm standard error of mean is shown in each chart. FC - fold-change. C - control samples (PBMCs from healthy volunteers). T0 - PBMCs obtained from patients before nephrectomy. T2 - PBMCs obtained from patients 12 months after surgery. $n$ - number of samples

database TMEM213 gene encodes a protein from the family of unknown function and average length of 154 aa, present in all eukaryotes. The Metaserver prediction suggests TMEM213 might contain two transmembrane (TM) helix segments (aa 7-26 and 71-91) with no tertiary homology to any protein crystal structures, currently available in RCSB PDB database. PSORT II classified TMEM213 as type 1a topology protein with one TM domain (aa 75-90). In contrast to Metaserver, PSORT II assigned aa 1-27 as a cleavable N-terminal signal peptide instead of TM domain (Additional file 7: Table S4). PSORT II NNCN Reinhardt's method for cytoplasmic/nuclear discrimination [52] scored TMEM213 as nuclear protein with reliability of 55.5. k-NN prediction for subcellular localization [56] determined TMEM213 to be connected to endoplasmic reticulum with probability of $44.4 \%$. Localizome analysis resulted in similar prediction to PSORT II (Fig. 5). Similar analyses were performed for the remaining 9 TMEMs as shown in Table 4.

No Pfam results were obtained for TMEM207, TMEM72 and TMEM116. TMEM61 was assigned as a member of TMEM61 family present in eukaryotes with no known function. TMEMs 45A and 45B were found to be DUF716 (Domain of Unknown Function 716) proteins, present in all metazoa, and predicted to modulate response to viral attack in plants. TMEM30B belongs to CDC50/LEM3 (ligand-effect modulator 3) family. LEM3 affects a downstream step of the glucocorticoid receptor pathway. RTP3 (TMEM7) was predicted to contain pairs of $\mathrm{CxxC}$ motifs possibly representing a multiple zinc-binding region, characteristic to zf-3CxxC family. SLC35G2 (TMEM22) on the other hand was recognized as EamA drug/metabolite transporter-like family member with two copies of EamA domain also known as AMAC (acyl-malonyl condensing enzyme) transporter.

In order to confirm known homologs identified by Pfam we analyzed the protein sequences with additional foldrecognition methods available at Metaserver Genesilico gateway. TMEM213, TMEM61, TMEM45B, TMEM30B TMEM72 and TMEM45A showed no homologous crystal structures deposited in RCSB PDB. However comparison, evaluation and ranking by the Pcons5 algorithm [46] at Metaserver denoted similarities of the remaining four TMEMs to known proteins. TMEM207 shared similarity with transmembrane protein stannin (PDB Id: 1ZZA), a small protein involved in inhibition of apoptosis) [59]. TMEM116 was inferred similar to human adrenoreceptor (PDB Id: 2RHL and 2R4R) and human adenoreceptor (PDB Id: 3EML). Similarly to Pfam analysis RTP3 homologs were found in a heterogeneous group of $\mathrm{Zn}$-binding proteins with conserved Zn-binding domain (PDB Id: 2CUP, 2DKT, 2E2Z and 3HCS) and SLC35G2 showed similarities to TM domain of the multidrug-resistance antiporter from E. coli 
Table 4 Detailed bioinformatic characteristics of TMEMs with topology and function predictions. ${ }^{1}$ - Metaserver prediction. ${ }^{2}$ - Localizome prediction, ${ }^{3}$ - PSORT II prediction. 4 - Functional data available; protein localized in Golgi apparatus, endosomes and lysosomes. Gl - GenInfo Identifier. TM - transmembrane helix segment. ER - endoplasmic reticulum. Mit - mitochondrial. Nuc - nuclear. Cyt - cytoplasmic. n/a - no result

\begin{tabular}{|c|c|c|c|c|c|c|c|c|c|c|}
\hline Property & TMEM213 & TMEM207 & TMEM61 & TMEM45B & TMEM30B & RTP3 & TMEM72 & TMEM116 & SLC35G2 & TMEM45A \\
\hline Full name & $\begin{array}{l}\text { transmembrane } \\
\text { protein } 213 \\
\text { precursor }\end{array}$ & $\begin{array}{l}\text { transmembrane } \\
\text { protein } 207 \\
\text { precursor }\end{array}$ & $\begin{array}{l}\text { transmembrane } \\
\text { protein } 61\end{array}$ & $\begin{array}{l}\text { transmembrane } \\
\text { protein } 45 \mathrm{~B}\end{array}$ & $\begin{array}{l}\text { transmembrane } \\
\text { protein } 30 B\end{array}$ & $\begin{array}{l}\text { receptor- } \\
\text { transporting } \\
\text { protein } 3\end{array}$ & $\begin{array}{l}\text { transmembrane } \\
\text { protein } 72\end{array}$ & $\begin{array}{l}\text { transmembrane } \\
\text { protein } 116 \\
\text { isoform } 1\end{array}$ & $\begin{array}{l}\text { solute carrier } \\
\text { family } 35 \\
\text { member G2 }\end{array}$ & $\begin{array}{l}\text { transmembrane } \\
\text { protein } 45 \mathrm{~A}\end{array}$ \\
\hline $\begin{array}{l}\text { Alternative } \\
\text { names }\end{array}$ & - & UNQ846 & - & - & CDC50B & $\begin{array}{l}\text { LTM1; } \\
\text { TMEM7 }\end{array}$ & $\begin{array}{l}\text { KSP37; } \\
\text { C10orf127; } \\
\text { bA285G1.3 }\end{array}$ & - & TMEM22 & DERP7 \\
\hline NP (NCBI) & 001078898.1 & 997199.1 & 872338.1 & 620143.1 & 001017970.1 & 113628.1 & 001116848.1 & 001180460.1 & 079522.2 & 060474.1 \\
\hline Gl & 146229352 & 46409276 & 32698902 & 20270331 & 63003930 & 13899263 & 183227675 & 302058299 & 148224156 & 8922243 \\
\hline $\begin{array}{l}\text { Protein } \\
\text { isoforms }\end{array}$ & 1 & 1 & 1 & 1 & 1 & 1 & 1 & 3 & 1 & 1 \\
\hline $\begin{array}{l}\text { Transcript } \\
\text { variants }\end{array}$ & 1 & 1 & 1 & 1 & 1 & 1 & 1 & 3 & 3 & 1 \\
\hline $\begin{array}{l}\text { Human length } \\
\text { (aa) }\end{array}$ & 107 & 146 & 210 & 275 & 351 & 232 & 275 & 337 & 412 & 275 \\
\hline Pfam & $\begin{array}{l}\text { TMEM213 family } \\
\text { in eukaryotes; } \\
154 \text { aa; unknown } \\
\text { function }\end{array}$ & n/a & $\begin{array}{l}\text { TMEM61 family } \\
\text { in eukaryotes; } \\
150-211 \text { aa; } \\
\text { unknown } \\
\text { function }\end{array}$ & $\begin{array}{l}\text { DUF716 family in } \\
\text { metazoa } \\
\text { (unknown } \\
\text { function) and } \\
\text { plants (response } \\
\text { to viral attack) }\end{array}$ & $\begin{array}{l}\text { CDC50/ LEM3 } \\
\text { (ligand-effect } \\
\text { modulator 3) } \\
\text { family in } \\
\text { glucocorticoid } \\
\text { receptor pathway }\end{array}$ & $\begin{array}{l}\text { zf-3CxxC } \\
\text { family with } \\
\text { zinc-binding } \\
\text { domain }\end{array}$ & $\mathrm{n} / \mathrm{a}$ & $\mathrm{n} / \mathrm{a}$ & $\begin{array}{l}\text { EamA drug/ } \\
\text { metabolite } \\
\text { transporter- } \\
\text { like family } \\
\text { with two } \\
\text { copies of } \\
\text { EamA } \\
\text { domain }\end{array}$ & $\begin{array}{l}\text { DUF716 family in } \\
\text { metazoa } \\
\text { (unknown } \\
\text { function) and } \\
\text { plants (response } \\
\text { to viral attack) }\end{array}$ \\
\hline $\begin{array}{l}\text { Tertiary } \\
\text { structure } \\
\text { prediction }\end{array}$ & $\mathrm{n} / \mathrm{a}$ & $\begin{array}{l}\text { Similar to TM } \\
\text { protein stannin } \\
\text { (PDB Id: 1zza) }\end{array}$ & $\mathrm{n} / \mathrm{a}$ & $\mathrm{n} / \mathrm{a}$ & $\mathrm{n} / \mathrm{a}$ & $\begin{array}{l}\text { Similar to Zn- } \\
\text { binding pro- } \\
\text { teins (PDB Id: } \\
\text { 2cup; } 2 \mathrm{dkt} ; \\
\text { 2e2z; 3hcs) }\end{array}$ & $\mathrm{n} / \mathrm{a}$ & $\begin{array}{l}\text { Similar to human } \\
\text { adrenoreceptor } \\
\text { (PDB Id: } 2 \text { rh1; } \\
\text { 2r4r) and human } \\
\text { adenoreceptor } \\
\text { (PDB Id: 3eml) }\end{array}$ & $\begin{array}{l}\text { Similar to } \\
\text { TM domain } \\
\text { of the } \\
\text { multidrug- } \\
\text { resistance } \\
\text { antiporter } \\
\text { from E. coli } \\
\text { EmrE (PDB } \\
\text { ld: } 3 \text { b5d) }\end{array}$ & $\mathrm{n} / \mathrm{a}$ \\
\hline TM domains & 1 & 1 & 2 & 7 & 2 & 1 & 4 & 3 & $10 \quad 10$ & 7 \\
\hline $\begin{array}{lll}1 & 2 & 3\end{array}$ & & & & & & & & & & \\
\hline $\begin{array}{l}\text { Predicted } \\
\text { topology }\end{array}$ & $\begin{array}{l}\text { Type 1a with cyt } \\
\text { tail (92 to 107) }\end{array}$ & $\begin{array}{l}\text { Type } 1 \text { a with cyt } \\
\text { tail (71 to } 146)\end{array}$ & Type 3a & Type 3b & Type 3a & $\begin{array}{l}\text { Type Nt with } \\
\text { cyt tail (1 to } \\
211 \text { ) }\end{array}$ & $\begin{array}{l}\text { Type } 2 \text { with cyt } \\
\text { tail ( } 1 \text { to } 94)\end{array}$ & Type 3a & Type 3a & Type 3a \\
\hline \multirow{2}{*}{$\begin{array}{l}\text { Cleavable N- } \\
\text { terminal signal } \\
\text { peptide }\end{array}$} & Yes ( 1 to 27$)$ & Yes (1 to 29) & No & No & No & No & No & Yes (1 to 50$)$ & No & No \\
\hline & Nuc (55.5) & Cyt (94.1) & Nuc (70.6) & Cyt (94.1) & Cyt (89) & Cyt (89) & Cyt (94.1) & Cyt (94.1) & Cyt (94.1) & Cyt (94.1) \\
\hline
\end{tabular}


Table 4 Detailed bioinformatic characteristics of TMEMs with topology and function predictions. ${ }^{1}$ - Metaserver prediction. ${ }^{2}$ - Localizome prediction, ${ }^{3}$ - PSORT II prediction. 4 - Functional data available; protein localized in Golgi apparatus, endosomes and lysosomes. Gl - GenInfo Identifier. TM - transmembrane helix segment. ER - endoplasmic reticulum. Mit - mitochondrial. Nuc - nuclear. Cyt - cytoplasmic. n/a - no result (Continued)

\begin{tabular}{|c|c|c|c|c|c|c|c|c|c|c|}
\hline \multicolumn{11}{|c|}{$\begin{array}{l}\text { Cytoplasmic/ } \\
\text { Nuclear } \\
\text { discrimination }{ }^{3}\end{array}$} \\
\hline $\begin{array}{l}\text { Subcellular } \\
\text { localization }^{3}\end{array}$ & ER (44.4 \%) & ER (44.4\%) & $\begin{array}{l}\text { Mit (39.1 \%) Nuc } \\
(34.8 \%)\end{array}$ & ER (66.7 \%) & $\begin{array}{l}\text { ER (39.1 \%) Mit } \\
(39.1 \%)\end{array}$ & Cyt (30.4 \%) & Mit (30.4 \%) & ER (43.5 \%) & $\mathrm{ER}^{4}(55.6 \%)$ & ER (77.8 \%) \\
\hline
\end{tabular}




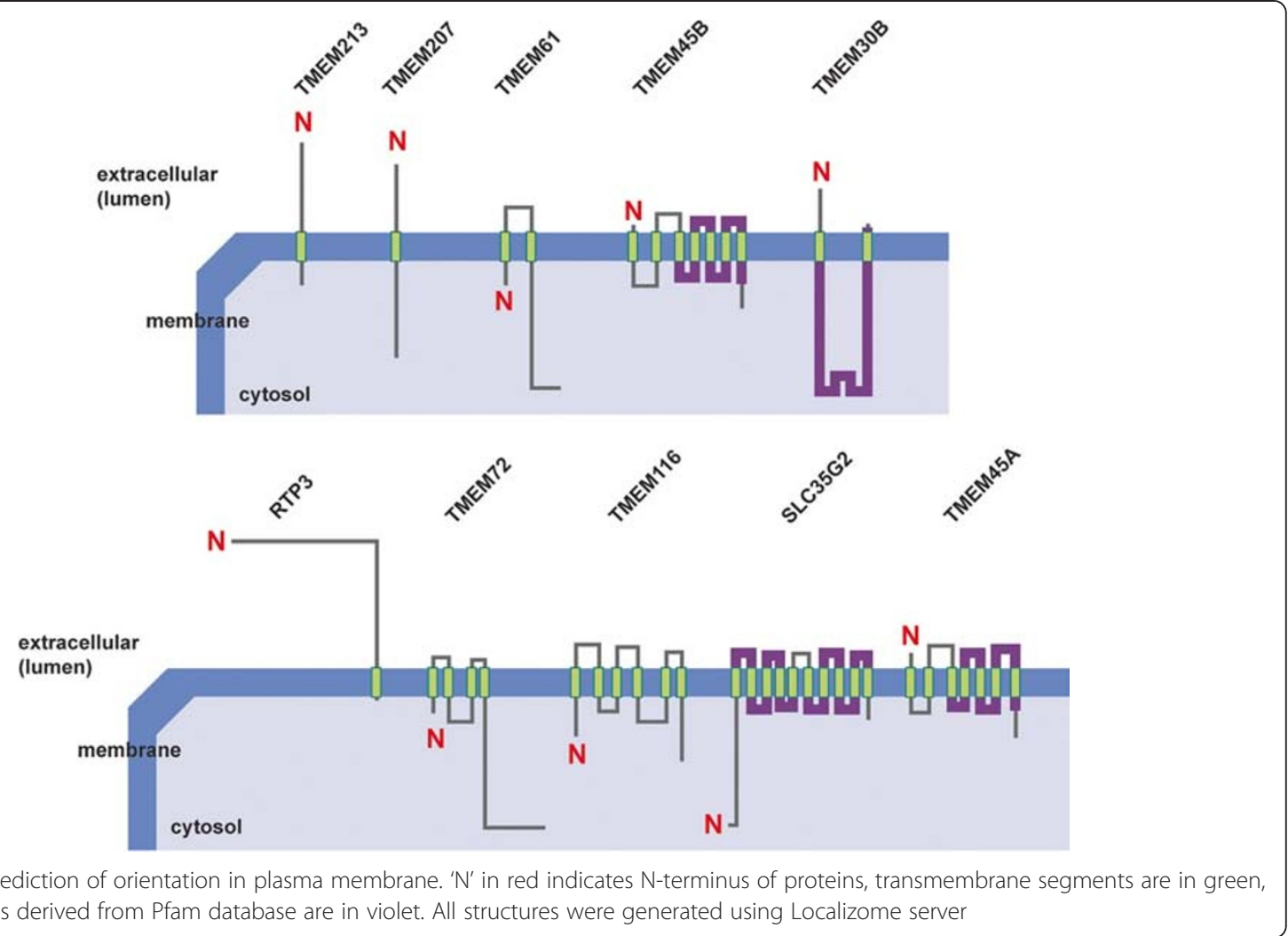

EmrE (PDB Id: 3B5days) was found. For RTP3 and SLC35G2 we found confirmation of Metaserver prediction with Pfam results.

Employing Singer's classification of proteins TMEMs have been sorted according to their predicted topology [50]. Majority of TMEMs are classified as type 3a transmembrane proteins (TMEM61, TMEM30B, TMEM116, SLC35G2 and TMEM45A). TMEM 45B is assigned as type 3b transmembrane protein, whereas TMEM72 - type 2 . TMEM213 and TMEM207 were grouped as type 1a with a cleavable signal sequence and one transmembrane segment. RTP3 is a protein with no cleavable signal sequence and one transmembrane segment near C-terminus (type $\mathrm{Nt}$ ).

Subcellular localization consensus analysis at PSORT II connected TMEM213, TMEM207, TMEM45B, TMEM116, SLC35G2 and TMEM45A with endoplasmic reticulum (with reliability of $44.4 \%$; $44.4 \%$; $66.7 \%$; $43.5 \%$; $55.6 \%$ and $77.8 \%$, respectively). RTP3 was found to be cytoplasmic (30.4 \%) and TMEM72 - mitochondrial (30.4 \%). For TMEM61 and TMEM30B we obtained inconclusive results: mitochondrial/nuclear (39.1 \%/34.8 \%) and ER/mitochondrial (39.1\%/39.1\%), respectively.

\section{Discussion}

Recently published meta-analysis study of differentially expressed genes in ccRCC led us to examine in detail the expression of ten genes encoding transmembrane proteins
(TMEMs) in tumor and PBMC samples derived from ccRCC patients [20]. TMEM protein family is characterized by a presence of putative transmembrane domains, as predicted by in silico methods, but limited functional data describing their detailed involvement in malignant transformation is available [29].

Majority of ccRCC deregulated TMEMs were assigned, according to Singer's classification, as type 3 transmembrane proteins characterized by multiple transmembrane domains in a single polypeptide chain [50]. This group was represented by TMEM61, TMEM30B, TMEM116 and TME M45B down-regulated in ccRCC tumors and up-regulated TMEM22 (SLC35G2) and TMEM45A. TMEM61 belongs to TMEM61 protein family of unknown function. TME M30B, predicted member of CDC50/LEM3 family of transcription regulators, facilitates a positive regulation of protein exit from endoplasmic reticulum in a cell $[60,61]$. Its' down-regulation was reported in advanced and recurrent samples of meningioma [11]. Similarly, we found more pronounced down-regulation in less differentiated tumors in our data set. TMEM116 showed tertiary structure similarity to human B2-adrenergic $\mathrm{G}$ protein-coupled receptor (GPCR) and human A2A adenosine receptor, found functionally deregulated in other cancer types and associated with tumor invasiveness and evasion of immune system [62-64]. Here we found TMEM116 expression to be a potential independent predictor of overall survival, as shown 
by Cox regression, with hazard ratio with each 2-fold increase of TMEM116 expression equal to 0.554. Cellular localization of the four down-regulated TMEMs was predicted as cytoplasmic, with TMEM116 to be likely of endoplasmic reticulum origin.

The up-regulated TMEM22 and TMEM45A encoded proteins were predicted to localize in endoplasmic reticulum. TMEM22 contains a domain similar to E.coli multidrug-resistance antiporter Emre, while TMEM45A (together with TMEM45B) was assigned to DUF716 protein family of unknown function in humans (Fig. 6). TMEM22 (SLC35G2) encodes a member G2 protein of solute carrier family 35 , a polytopic transmembrane protein found in Golgi apparatus, endosomes and lysosomes $[65,66]$ possibly involved in nucleoside-sugar transport [67]. Differential regulation in SLC35G2 expression in intrahepatic cholangiocarcinoma [68] and promoter hypermethylation of SLC35G2 in melanoma were previously reported [69]. Dobashi et al. found upregulation of SLC35G2 in Caki-1 and Caki-2 RCC cell lines and tumor samples; additionally, siRNA silencing of SLC35G2 diminished cell growth in those cell lines, suggesting its potential involvement in cancer progression and development [29]. Here, we also observe up-regulation of $S L C 35 G 2$ expression in our tumor set. Additionally, we find SLC35G2 expression to be upregulated in PBMCs collected from patients a year postnephrectomy, as compared to PBMCs of healthy volunteers. This finding is very promising, although it requires additional conformation in an independent cohort.
TMEM45A is a substrate for ubiquitin ligase and therefore can be degraded in proteasome [70], similarly to TMEM45B, which interacts with ubiquitin-conjugating enzyme E2G 2 [71]. Lee et al. observed that progression of ductal carcinoma in situ to invasive breast cancer in xenograft models increased dramatically when TMEM45A expression was suppressed [72]. TMEM45B down-regulation was detected in squamous lung cell carcinoma, likely due to increased expression of miRNA targeting TMEM45B gene [73]. Paulo et al. suggests TMEM45B regulation by ERG and $E T V 1$ transcription factors in prostate cancer cell lines [74]. TMEM45B interacts with tubulointerstitial nephritis antigen (TINAG), which is thought to be involved in FAK/ PI3K/Akt-mediated apoptosis [75]. TINAG expression is also down-regulated in ccRCC, as shown by microarray experiments [17]. We found that expression decrease of TMEM45B may be associated with organ-confined tumors, as shown by $t$-test.

Two proteins: TMEM213 and TMEM207 were assigned to transmembrane protein type $1 \mathrm{a}$ with a cleavable signal sequence [50]. Both TMEM213 and TME M207 genes were the most down-regulated in our ccRCC sample set. TMEM213 was predicted to be localized in ER and in silico analyses demonstrated existence of TMEM213 protein family with unknown function. TMEM207, with predicted cytoplasmic localization, showed a limited homology to Stannin (SNN), likely involved in mitochondria-mediated apoptosis [76, 77]. Although hierarchical clustering showed that downregulation of both TMEM213 and TMEM207 genes,

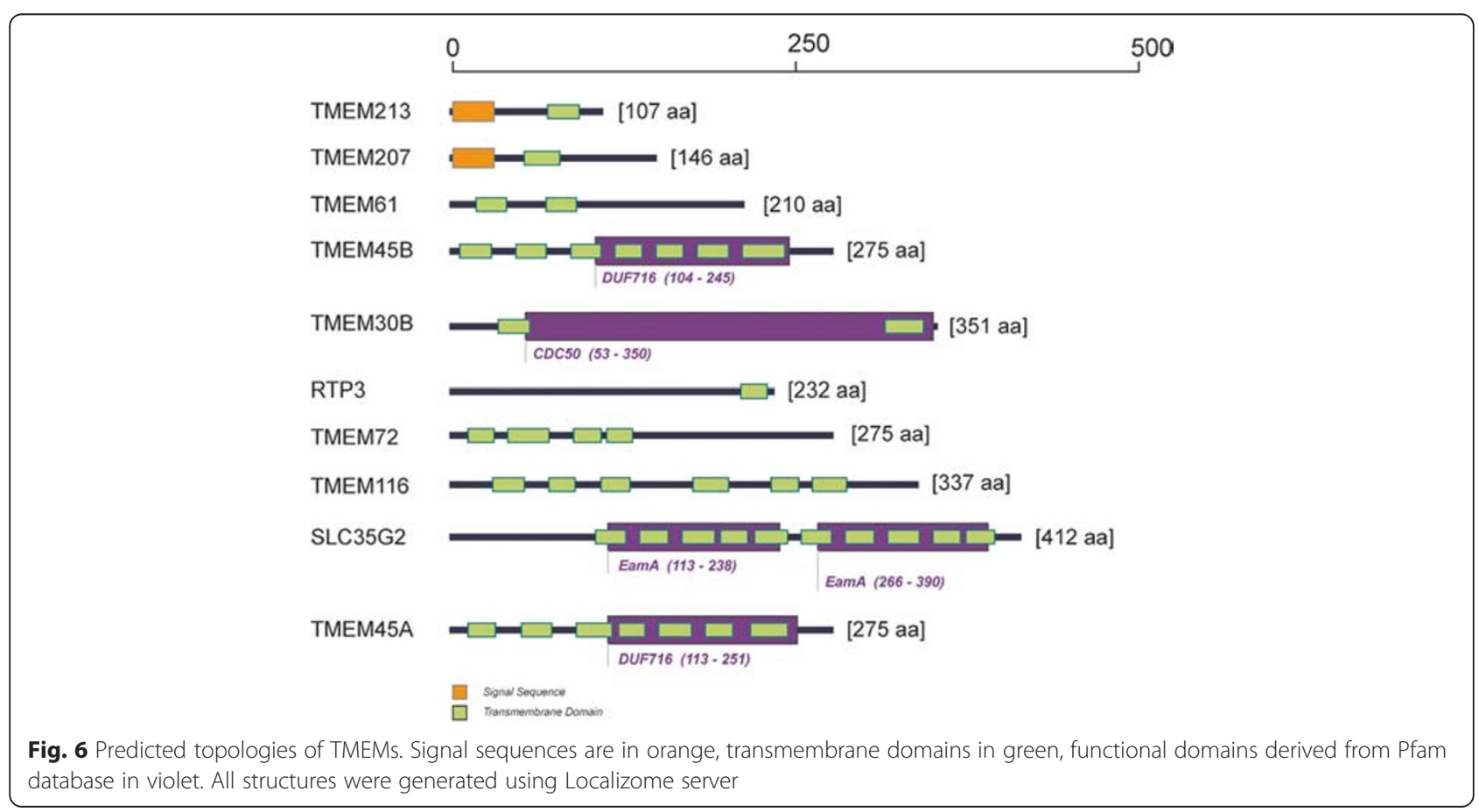


together with TMEM72 and TMEM116 could characterize more advanced ccRCC tumors, these observations require additional confirmation.

TMEM72, classified as a type 2 transmembrane protein, is predicted to be localized in mitochondria, with no similarities to known proteins. We found that strong TMEM72 down-regulation could be associated with metastases, as revealed by the $t$-test and univariate and multivariate logistic regression. Similar trend is observed in more advanced tumors, as showed by hierarchical clustering.

Previously RTP3 (TMEM7) was classified as type NT transmembrane protein with ER retention signal, is predicted to function in endoplasmic reticulum (ER) membrane $[78,79]$. RTP3 showed similarity to $\mathrm{zf}-3 \mathrm{CxxC}$ transcription regulators family, previously reported to be involved in renal cell carcinoma [80, 81]. Zhou et al. show that down-regulation or inactivation of RTP3 was detected in $85 \%$ of primary hepatocellular carcinomas and in $33 \%$ of hepatocellular carcinoma cell lines, likely due to promoter hypermethylation [82]. Although RTP3 was significantly down-regulated in our tumor set, we did not observe any relevance of this down-regulation in respect to clinical and molecular tumor characteristics.

Interestingly, five of analyzed TMEMs were predicted to have ER membrane localization signals. Endoplasmic reticulum stress has a profound effect on cancer cell proliferation and survival in almost all types of cancer, including RCC [62]. Duivenvoorden et al. found that endoplasmic reticulum protein - ERp46 - expression is elevated in ccRCC [83]. Moreover, von Roemeling and colleagues report that inhibition of aberrant stearoylCoA desaturase 1 (SCD1) expression attenuates cell proliferation and induces apoptosis in ccRCC cells via the induction of endoplasmic reticulum stress response signaling [84]. Although our findings require further validation, we show that TMEM proteins predicted to be localized in ER (i.e., SLC35G2, TMEM45A, TME M45B, TMEM116, TMEM207 and TMEM213) can be potentially involved in ccRCC pathogenesis.

\section{Conclusions}

The massive down-regulation of expression of TMEM family members suggests their significant involvement in the pathogenesis of ccRCC. We found that downregulation of all the TMEMs is most pronounced in advanced tumors and linked their deregulation to metastatic tumors, high Fuhrman grade and disease course. Topology and localization analysis classified majority of the TMEMs as type 3 and type 1 transmembrane proteins, with predicted localization in endoplasmic reticulum, prominently, supporting the involvement of ER proteins in ccRCC pathogenesis.

\section{Additional files}

Additional file 1: Table S2. Clinical characteristics. Detailed clinical characteristics of patients involved in the study at the time of nephrectomy.

Additional file 2: Table S3. Follow-up characteristics. Detailed characteristics of patients' follow-up. Time to progression - time from nephrectomy to the latest follow-up (in months) or to the follow-up where progression occurred. Time to death - time from nephrectomy to the latest follow-up (in months) or to the death date. In 'Progression' and 'Death' columns 0 means no progression or death (censored case), 1 means that the disease progressed (type of progression is summarized in 'Type of progression' column) or patient died from the disease. N/A - data not available.

Additional file 3: Table S1. Primer sequences used for GPCR TMEM expression measurements.

Additional file 4: Figure S1. TMEM expression in metastatic and non-metastatic tumors. Average $\log _{2}$ relative expression data in each sample group \pm standard error of mean is shown in each chart. FC - fold-change. $n$ - number of samples. M0 - non-metastatic cCRCC. M1 - metastatic cCRCC tissues. q - p-values adjusted for multiple comparisons using BenjaminiHochberg correction.

Additional file 5: Figure S2. TMEM expression in tumors at early and late stage of the disease, as assessed by TNM staging system. Average $\log _{2}$ relative expression data in each sample group \pm standard error of mean is shown in each chart. FC - fold-change. $n$ - number of samples. pT1/pT2 - organconfined tumors, as assessed by TNM staging system. pT3/pT4 - advanced tumors, as assessed by TNM staging system. $q$ - p-values adjusted for multiple comparisons using Benjamini-Hochberg correction.

Additional file 6: Figure S3. TMEM expression in tumors comprised of well differentiated or undifferentiated cells, as assessed by Fuhrman grading system. Average $\log _{2}$ relative expression data in each sample group \pm standard error of mean is shown in each chart. FC - fold-change. $n$ - number of samples. G1/G2 - low Fuhrman grade samples. G3/G4 - high Furhman grade samples. $\mathrm{q}$ - $\mathrm{p}$-values adjusted for multiple comparisons using Benjamini-Hochberg correction.

Additional file 7: Table S4. Colocalization of topology and function prediction for TMEM213 at Metaserver Genesilico gateway . Colocalization of topology and function prediction for TMEM213 at Metaserver Genesilico gateway. TM helices were superimposed to the results from PSORT II and Localizome.

\section{Abbreviations}

cCRCC: Clear cell renal cell carcinoma; TMEM(s): Transmembrane proteins; ER: Endoplasmic reticulum; qPCR: Quantitative polymerase chain reaction; RTP3: Receptor (chemosensory) transporter protein 3; SLC35G2: Solute carrier family 35, member G2; TMEM30B: Transmembrane protein 30B;

TMEM45A: Transmembrane protein 45A; TMEM45B: Transmembrane protein 45B; TMEM61: Transmembrane protein 61; TMEM72: Transmembrane protein 72; TMEM116: Transmembrane protein 116; TMEM207: Transmembrane protein 207; TMEM213: Transmembrane protein 213; RCC: Renal cel carcinoma; VHL: Von Hippel-Lindau syndrome/protein; LOH: Loss of heterozygosity; KNG1: Kininogen 1; MT1A: Metallothionein 1A; PI3K: Phosphatidylinositol-4,5-bisphosphate 3-kinase; AKT: v-Akt murine thymoma viral oncogene homolog; mTOR: Mechanistic target of rapamycin; MTOR: Mechanistic target of rapamycin (serine/threonine kinase); PTEN: Phosphatase and tensin homolog; PIK3CA: Phosphatidylinositol-4,5bisphosphate 3-kinase, catalytic subunit alpha; TMEM176: Transmembrane protein 176; TMEM25: Transmembrane protein 25;

TMEM127: Transmembrane protein 127; ca.: circa; ANO1: Anoctamin 1, calcium activated chloride channel; TMEM123: Transmembrane protein 123; TMEM165: Transmembrane protein 165;

TMEM181: Transmembrane protein 181; TMEM173: Transmembrane protein 173; RT-PCR: Reverse transcription - polymerase chain reaction; RAB37: RAB37, member RAS oncogene family; HIF1A: Hypoxia inducible factor 1, alpha subunit; EPAS1: Endothelial PAS domain protein 1; PBMCs: Peripheral blood mononuclear cells; CDNA: Complementary DNA; CNRQ: Calculated normalized relative quantity; ACTB: beta-actin; 
ANOVA: one-way analysis of variance; AIC: Akaike Information Criterion; VIF: Variance inflation factor; q-value: $\mathrm{p}$-value corrected by Benjamini-Hochberg method; aa: amino acid; HMM: Hidden Markov model; RSA: Relative solvent accessibility of amino acid; RCSB PDB: Research Collaboratory for Structural Bioinformatics Protein Data Bank; FC: Fold-change; N: number of samples/cases; pT: primary tumor stage; G: Fuhrman grade; r: Pearson correlation coefficient; TNM: Tumor Node Metastases staging system; OR: odds ratio; $95 \%$ Cl: $95 \%$ confidence interval; ROC AUC: Receiver Operating Characteristics Area Under Curve; HR: Hazard ratio; T0: Time-point before nephrectomy; T2: Time-point one year post-nephrectomy; TM: Trans-membrane; DUF716: Domain of unknown function 716; LEM3: Ligand-effect modulator 3; EamA: O-acetyl-serine/ cysteine export gene in E. coli; AMAC: Acyl-malonyl condensing enzyme; GPCR: G-protein coupled receptor; ERG: Ets avian erythroblastosis virus E26 oncogene homolog; ETV1: Ets variant 1; FAK: protein tyrosine kinase 2; TMT: Trimethylin; SNN: Stannin; ERK: Mitogen-activated protein kinase 1; ERp46: Thioredoxin domain containing 5; SCD1: Stearoyl-Coenzyme A desaturase 1.

\section{Competing interests}

The authors declare that they have no competing interests.

\section{Authors' contributions}

TW performed TMEMs expression measurement, statistical analyses and wrote the first draft of the manuscript, MS performed bioinformatic analyses and contributed to the manuscript, WC, Al and ZK were involved in patient material collection (tumor and blood samples), RG participated in design of the study and helped to draft the manuscript, EZ performed qPCR expression measurement of VHL, HIFIA and EPAS1, JS and JP participated in qPCR experiments, HB made substantial corrections of the manuscript and participated in study design, JW orchestrated, designed and coordinated the study and contributed and supervised the preparation of the manuscript. All authors read and approved the final manuscript.

\section{Acknowledgements}

This publication was supported by grant no. UMO-2012/05/N/NZ2/00635 funded by National Science Center (project leader: TW), by grant no. 502-1402226365-50333 funded by Poznan University of Medical Sciences (project leader: WC) and by FOCUS program no. 3/2008 funded by Polish Foundation of Science (project leader: JW). TW and MS are scholarship holders within the project "Scholarship support for PH.D. students specializing in majors strategic for Wielkopolska's development", Sub-measure 8.2.2 Human Capital Operational Programme, co-financed by European Union under the European Social Fund. The qPCR analysis was performed in Genome Analysis Laboratory funded by National Multidisciplinary Laboratory of Functional Nanomaterials NanoFun nr POIG.02.02.00-00-025/09 (Innovative Economy Operational Programme, Priority Axis 2: R\&D Infrastructure, Action 2.2: Support of Formation of Common Research Infrastructure of Scientific Units). Adam Mickiewicz University, Faculty of Biology is a member of KNOW Poznan RNA Centre (no. 01/KNOW2/2014).

\section{Author details}

'Laboratory of High Throughput Technologies, Institute of Molecular Biology and Biotechnology, Faculty of Biology, Adam Mickiewicz University, Umultowska 89, 61-614 Poznan, Poland. '2Department of Human Molecular Genetics, Institute of Molecular Biology and Biotechnology, Faculty of Biology, Adam Mickiewicz University, Umultowska 89, 61-614 Poznan, Poland. ${ }^{3}$ Department of Urology and Urological Oncology, Poznan University of Medical Sciences, Szwajcarska 3, 61-285 Poznan, Poland. ${ }^{4}$ Department of Nephrology and Hypertension, University Medical Center, Postbus 85500, 3508 GA Utrecht, Netherlands.

Received: 8 October 2014 Accepted: 1 July 2015 H.t.

\section{References}

1. Rydzanicz M, Wrzesinski T, Bluyssen HA, Wesoly J. Genomics and epigenomics of clear cell renal cell carcinoma: recent developments and potential applications. Cancer Lett. 2013;341(2):111-26.

2. Rechsteiner MP, von Teichman A, Nowicka A, Sulser T, Schraml P, Moch H. $\mathrm{VHL}$ gene mutations and their effects on hypoxia inducible factor HIFalpha: identification of potential driver and passenger mutations. Cancer Res. 2011;71(16):5500-11.

3. Lipworth L, Tarone RE, McLaughlin JK. The epidemiology of renal cell carcinoma. J Urol. 2006;176(6 Pt 1):2353-8.

4. Kim WY, Kaelin WG. Role of VHL gene mutation in human cancer. J Clin Oncol. 2004;22(24):4991-5004.

5. Nelson EC, Evans CP, Lara Jr PN. Renal cell carcinoma: current status and emerging therapies. Cancer Treat Rev. 2007;33(3):299-313.

6. Ong KR, Woodward ER, Killick P, Lim C, Macdonald F, Maher ER. Genotype-phenotype correlations in von Hippel-Lindau disease. Hum Mutat. 2007;28(2):143-9.

7. Takahashi M, Rhodes DR, Furge KA, Kanayama H, Kagawa S, Haab BB, et al. Gene expression profiling of clear cell renal cell carcinoma: gene identification and prognostic classification. Proc Natl Acad Sci U S A. 2001;98(17):9754-9.

8. Sato Y, Yoshizato T, Shiraishi Y, Maekawa S, Okuno Y, Kamura T, et al. Integrated molecular analysis of clear-cell renal cell carcinoma. Nat Genet. 2013;45(8):860-7.

9. Cuajungco MP, Podevin W, Valluri VK, Bui Q, Nguyen VH, Taylor K. Abnormal accumulation of human transmembrane (TMEM)-176A and $176 \mathrm{~B}$ proteins is associated with cancer pathology. Acta Histochem. 2012;114(7):705-12.

10. Hrasovec S, Hauptman N, Glavac D, Jelenc F, Ravnik-Glavac M. TMEM25 is a candidate biomarkermethylated and down-regulated in colorectal cancer. Dis Markers. 2013;34(2):93-104

11. Perez-Magan E, Campos-Martin Y, Mur P, Fiano C, Ribalta T, et al. Genetic alterations associated with progression and recurrence in meningiomas. J Neuropathol Exp Neurol. 2012;71(10):882-93.

12. Abermil N, Guillaud-Bataille $M$, Burnichon $N$, Venisse A, Manivet $P$, Guignat $L$, et al. TMEM127 screening in a large cohort of patients with pheochromocytoma and/or paraganglioma. J Clin Endocrinol Metab. 2012;97(5):E805-9.

13. Beroukhim R, Brunet JP, Di Napoli A, Mertz KD, Seeley A, Pires MM, et al. Patterns of gene expression and copy-number alterations in von-hippel lindau disease-associated and sporadic clear cell carcinoma of the kidney. Cancer Res. 2009;69(11):4674-81.

14. Cifola I, Spinelli R, Beltrame L, Peano C, Fasoli E, Ferrero S, et al. Genome-wide screening of copy number alterations and $\mathrm{LOH}$ events in renal cell carcinomas and integration with gene expression profile. Mol Cancer. 2008;7:6.

15. Gumz ML, Zou H, Kreinest PA, Childs AC, Belmonte LS, LeGrand SN, et al. Secreted frizzled-related protein 1 loss contributes to tumor phenotype of clear cell renal cell carcinoma. Clinical cancer research : an official journal of the American Association for Cancer Research. 2007;13(16):4740-9.

16. Jones J, Otu H, Spentzos D, Kolia S, Inan M, Beecken WD, et al. Gene signatures of progression and metastasis in renal cell cancer. Clinical cancer research : an official journal of the American Association for Cancer Research. 2005;11(16):5730-9.

17. Tun HW, Marlow LA, von Roemeling CA, Cooper SJ, Kreinest P, Wu K, et al. Pathway signature and cellular differentiation in clear cell renal cell carcinoma. PLoS One. 2010;5(5), e10696.

18. Wang $Y$, Roche $O$, Yan MS, Finak G, Evans AJ, Metcalf $J$, et al. Regulation of endocytosis via the oxygen-sensing pathway. Nat Med. 2009;15(3):319-24.

19. Dalgliesh GL, Furge K, Greenman C, Chen L, Bignell G, Butler A, et al. Systematic sequencing of renal carcinoma reveals inactivation of histone modifying genes. Nature. 2010;463(7279):360-3.

20. Zodro E, Jaroszewski M, Ida A, Wrzesinski T, Kwias Z, Bluyssen H, Wesoly J. FUT11 as a potential biomarker of clear cell renal cell carcinoma progression based on meta-analysis of gene expression data. Tumour Biol. 2014:35(3):2607-17

21. Fuller CM. Time for TMEM? J Physiol. 2012;590(Pt 23):5931-2.

22. Ma F, Zhang C, Prasad KV, Freeman GJ, Schlossman SF. Molecular cloning of Porimin, a novel cell surface receptor mediating oncotic cell death. Proc Natl Acad Sci U S A. 2001;98(17):9778-83.

23. Foulquier F, Amyere M, Jaeken J, Zeevaert R, Schollen E, Race V, et al. TMEM165 deficiency causes a congenital disorder of glycosylation. Am J Hum Genet. 2012;91(1):15-26.

24. Carette JE, Guimaraes CP, Varadarajan M, Park AS, Wuethrich I, Godarova A, et al. Haploid genetic screens in human cells identify host factors used by pathogens. Science. 2009;326(5957):1231-5.

25. Ishikawa H, Barber GN. STING is an endoplasmic reticulum adaptor that facilitates innate immune signalling. Nature. 2008;455(7213):674-8.

26. Kholodnyuk ID, Kozireva S, Kost-Alimova M, Kashuba V, Klein G, Imreh S. Down regulation of $3 p$ genes, LTF, SLC38A3 and DRR1, upon growth of 
human chromosome 3-mouse fibrosarcoma hybrids in severe combined immunodeficiency mice. Int J Cancer. 2006;119(1):99-107.

27. Togashi A, Katagiri T, Ashida S, Fujioka T, Maruyama O, Wakumoto Y, et al. Hypoxiainducible protein 2 (HIG2), a novel diagnostic marker for renal cell carcinoma and potential target for molecular therapy. Cancer Res. 2005;65(11):4817-26.

28. Hirota E, Yan L, Tsunoda T, Ashida S, Fujime M, Shuin T, et al. Genome-wide gene expression profiles of clear cell renal cell carcinoma: identification of molecular targets for treatment of renal cell carcinoma. Int J Oncol. 2006;29(4):799-827.

29. Dobashi S, Katagiri T, Hirota E, Ashida S, Daigo Y, Shuin T, et al. Involvement of TMEM22 overexpression in the growth of renal cell carcinoma cells. Oncol Rep. 2009;21(2):305-12

30. R: A language and environment for statistical computing. R Foundation for Statistical Computing [http://www.R-project.org/]

31. Akaike H. A new look at the statistical model identification. IEEE Trans Autom Control. 1974;19(6):716-23.

32. leaps: regression subset selection. R package version 2.9 [http://CRAN.Rproject.org/package=leaps]

33. Friedman J, Hastie T, Tibshirani R. Regularization Paths for Generalized Linear Models via Coordinate Descent. J stat softw. 2010;33(1):1-22.

34. Friedman J, Hastie T, Tibshirani R. Regularization paths for generalized linear models via coordinate descent. J stat softw. 2010;33(1):1.

35. O'brien RM. A caution regarding rules of thumb for variance inflation factors. Qual Quant. 2007;41(5):673-90.

36. Gray RJ. Flexible methods for analyzing survival data using splines, with applications to breast cancer prognosis. J Am Stat Assoc. 1992;87(420):942-51.

37. Harrell FE. Regression modeling strategies: with applications to linear models, logistic regression, and survival analysis: Springer. 2001. http://link.springer.com/book/10.1007\%2F978-1-4757-3462-1

38. Le Cessie S, Van Houwelingen J. Ridge estimators in logistic regression. Appl stat. 1992;191-201.

39. Verweij PJ, Van Houwelingen HC. Penalized likelihood in cox regression. Stat Med. 1994;13(23-24):2427-36.

40. Verweij PJ, van Houwelingen HC. Time-dependent effects of fixed covariates in Cox regression. Biometrics. 1995;1550-1556.

41. Simon N, Friedman J, Hastie T, Tibshirani R. Regularization paths for Cox's proportional hazards model via coordinate descent. J stat softw. 2011;39(5):1-13.

42. Grambsch PM, Therneau TM. Proportional hazards tests and diagnostics based on weighted residuals. Biometrika. 1994;81(3):515-26.

43. Benjamini $Y$, Hochberg Y. Controlling the false discovery rate: a practical and powerful approach to multiple testing. J R Stat Soc Ser B Methodol. 1995;289-300.

44. Finn RD, Bateman A, Clements J, Coggill P, Eberhardt RY, Eddy SR, et al. Pfam: the protein families database. Nucleic Acids Res. 2014;42(Database issue):D222-30.

45. Kurowski MA, Bujnicki JM. GeneSilico protein structure prediction meta-server. Nucleic Acids Res. 2003;31(13):3305-7.

46. Wallner B, Elofsson A. Pcons5: combining consensus, structural evaluation and fold recognition scores. Bioinformatics. 2005;21 (23):4248-54.

47. Nakai K, Kanehisa M. A knowledge base for predicting protein localization sites in eukaryotic cells. Genomics. 1992;14(4):897-911.

48. McGeoch DJ. On the predictive recognition of signal peptide sequences. Virus Res. 1985;3(3):271-86.

49. von Heijne G. A new method for predicting signal sequence cleavage sites. Nucleic Acids Res. 1986;14(11):4683-90.

50. Singer SJ. The structure and insertion of integral proteins in membranes. Annu Rev Cell Biol. 1990;6:247-96.

51. Klein $\mathrm{P}$, Kanehisa M, DeLisi C. The detection and classification of membranespanning proteins. Biochim Biophys Acta. 1985;815(3):468-76.

52. Reinhardt A, Hubbard T. Using neural networks for prediction of the subcellular location of proteins. Nucleic Acids Res. 1998:26(9):2230-6.

53. McNew JA, Goodman JM. The targeting and assembly of peroxisomal proteins: some old rules do not apply. Trends Biochem Sci. 1996;21 (2):54-8.

54. Teasdale RD, Jackson MR. Signal-mediated sorting of membrane proteins between the endoplasmic reticulum and the golgi apparatus. Annu Rev Cell Dev Biol. 1996;12:27-54.

55. Stack JH, Horazdovsky B, Emr SD. Receptor-mediated protein sorting to the vacuole in yeast: roles for a protein kinase, a lipid kinase and GTP-binding proteins. Annu Rev Cell Dev Biol. 1995;11:1-33.

56. Horton P. Nakai K. Better prediction of protein cellular localization sites with the $k$ nearest neighbors classifier. Proceedings / International Conference on Intelligent Systems for Molecular Biology; ISMB International Conference on Intelligent Systems for Molecular Biology. 1997;5:147-52.
57. Lee S, Lee B, Jang I, Kim S, Bhak J. Localizome: a server for identifying transmembrane topologies and TM helices of eukaryotic proteins utilizing domain information. Nucleic acids res. 2006;34:W99-103. Web Server issue.

58. Kall L, Krogh A, Sonnhammer EL. A combined transmembrane topology and signal peptide prediction method. J Mol Biol. 2004;338(5):1027-36.

59. Buck-Koehntop BA, Mascioni A, Buffy JJ, Veglia G. Structure, dynamics, and membrane topology of stannin: a mediator of neuronal cell apoptosis induced by trimethyltin chloride. J Mol Biol. 2005;354(3):652-65.

60. Collart MA, Struhl K. CDC39, an essential nuclear protein that negatively regulates transcription and differentially affects the constitutive and inducible HIS3 promoters. EMBO J. 1993;12(1):177-86.

61. Takatsu H, Baba K, Shima T, Umino H, Kato U, Umeda M, et al. ATP9B, a P4-ATPase (a putative aminophospholipid translocase), localizes to the trans-Golgi network in a CDC50 protein-independent manner. J Biol Chem. 2011;286(44):38159-67.

62. Clarke HJ, Chambers JE, Liniker E, Marciniak SJ. Endoplasmic reticulum stress in malignancy. Cancer Cell. 2014;25(5):563-73.

63. Dorsam RT, Gutkind JS. G-protein-coupled receptors and cancer. Nat Rev Cancer. 2007;7(2):79-94.

64. Ohta A, Gorelik E, Prasad SJ, Ronchese F, Lukashev D, Wong MK, et al. A2A adenosine receptor protects tumors from antitumor T cells. Proc Natl Acad Sci U S A. 2006;103(35):13132-7.

65. Mehrle A, Rosenfelder H, Schupp I, del Val C, Arlt D, Hahne F, et al. The LIFEdb database in 2006. Nucleic Acids Res. 2006;34(Database issue):D415-8.

66. Simpson JC, Wellenreuther R, Poustka A, Pepperkok R, Wiemann S. Systematic subcellular localization of novel proteins identified by large-scale CDNA sequencing. EMBO Rep. 2000;1(3):287-92.

67. Ishida N, Kawakita M. Molecular physiology and pathology of the nucleotide sugar transporter family (SLC35). Pflugers Arch: Eur j physiol. 2004;447(5):768-75.

68. Wang AG, Yoon SY, Oh JH, Jeon YJ, Kim M, Kim JM, et al. Identification of intrahepatic cholangiocarcinoma related genes by comparison with normal liver tissues using expressed sequence tags. Biochem Biophys Res Commun. 2006;345(3):1022-32.

69. Furuta J, Nobeyama Y, Umebayashi Y, Otsuka F, Kikuchi K, Ushijima T. Silencing of Peroxiredoxin 2 and aberrant methylation of $33 \mathrm{CpG}$ islands in putative promoter regions in human malignant melanomas. Cancer Res. 2006;66(12):6080-6.

70. Povlsen LK, Beli P, Wagner SA, Poulsen SL, Sylvestersen KB, Poulsen JW, et al. Systems-wide analysis of ubiquitylation dynamics reveals a key role for PAF15 ubiquitylation in DNA-damage bypass. Nat Cell Biol. 2012;14(10):1089-98.

71. Wang J, Huo K, Ma L, Tang L, Li D, Huang X, et al. Toward an understanding of the protein interaction network of the human liver. Mol Syst Biol. 2011;7:536.

72. Lee S, Stewart S, Nagtegaal I, Luo J, Wu Y, Colditz G, et al. Differentially expressed genes regulating the progression of ductal carcinoma in situ to invasive breast cancer. Cancer Res. 2012;72(17):4574-86.

73. Molina-Pinelo S, Gutierrez G, Pastor MD, Hergueta M, Moreno-Bueno G, Garcia-Carbonero R, et al. MicroRNA-Dependent Regulation of Transcription in Non-Small Cell Lung Cancer. PLoS One. 2014;9(3), e90524.

74. Paulo P, Ribeiro FR, Santos J, Mesquita D, Almeida M, Barros-Silva JD, et al. Molecular subtyping of primary prostate cancer reveals specific and shared target genes of different ETS rearrangements. Neoplasia. 2012;14(7):600-11.

75. Xie P, Kondeti VK, Lin S, Haruna Y, Raparia K, Kanwar YS. Role of extracellular matrix renal tubulo-interstitial nephritis antigen (TINag) in cell survival utilizing integrin (alpha)vbeta3/focal adhesion kinase (FAK)/phosphatidylinositol 3-kinase (PI3K)/protein kinase B-serine/threonine kinase (AKT) signaling pathway. J Biol Chem. 2011;286(39):34131-46.

76. Billingsley ML, Yun J, Reese BE, Davidson CE, Buck-Koehntop BA, Veglia G. Functional and structural properties of stannin: roles in cellular growth, selective toxicity, and mitochondrial responses to injury. J Cell Biochem. 2006;98(2):243-50.

77. Davidson CE, Reese BE, Billingsley ML, Yun JK. Stannin, a protein that localizes to the mitochondria and sensitizes $\mathrm{NIH}-3 \mathrm{~T} 3$ cells to trimethyltin and dimethyltin toxicity. Mol Pharmacol. 2004;66(4):855-63.

78. Behrens M, Bartelt J, Reichling C, Winnig M, Kuhn C, Meyerhof W. Members of RTP and REEP gene families influence functional bitter taste receptor expression. J Biol Chem. 2006;281 (29):20650-9.

79. Kiss H, Yang Y, Kiss C, Andersson K, Klein G, Imreh S, et al. The transcriptional map of the common eliminated region 1 (C3CER1) in 3p21.3. European journal of human genetics : EJHG. 2002;10(1):52-61. 
80. Lahiri SK, Zhao J. Kruppel-like factor 8 emerges as an important regulator of cancer. Am j transl res. 2012;4(3):357-63.

81. Varrault A, Ciani E, Apiou F, Bilanges B, Hoffmann A, Pantaloni C, et al. hZAC encodes a zinc finger protein with antiproliferative properties and maps to a chromosomal region frequently lost in cancer. Proc Natl Acad Sci U S A. 1998;95(15):8835-40.

82. Zhou X, Popescu NC, Klein G, Imreh S. The interferon-alpha responsive gene TMEM7 suppresses cell proliferation and is downregulated in human hepatocellular carcinoma. Cancer Genet Cytogenet. 2007;177(1):6-15.

83. Duivenvoorden WC, Paschos A, Hopmans SN, Austin RC, Pinthus JH. Endoplasmic reticulum protein ERp46 in renal cell carcinoma. PLoS One. 2014;9(3), e90389.

84. von Roemeling CA, Marlow LA, Wei JJ, Cooper SJ, Caulfield TR, Wu K, et al. Stearoyl-CoA desaturase 1 is a novel molecular therapeutic target for clear cell renal cell carcinoma. Clinical cancer research : an official journal of the American Association for Cancer Research. 2013;19(9):2368-80.

\section{Submit your next manuscript to BioMed Central and take full advantage of:}

- Convenient online submission

- Thorough peer review

- No space constraints or color figure charges

- Immediate publication on acceptance

- Inclusion in PubMed, CAS, Scopus and Google Scholar

- Research which is freely available for redistribution 